\title{
Optimization of Dynamic Source Routing Protocol with Effective Resource Management
}

\author{
Lokesh Kumar Laddhani \\ Institute of Computer Science \\ Vikram University, Ujjain
}

\author{
Umesh Kumar Singh \\ Institute of Computer Science \\ Vikram University, Ujjain
}

\begin{abstract}
This paper proposes optimization of DSR (Dynamic Source Routing) protocol in aspect of cache size and cache expiry time to meet Quality of Service requirements between source and destination node pairs. The nodes are assumed to use a TDMA (Time Division Multiple Access) based MAC (Medium Access Control) layer. Given a set of Q o S requirements, a set of wireless nodes and their initial positions, the goal is to find a topology of the nodes by adjusting the transmitting power, which will meet the QoS requirements under the presence of interference and at the same time minimize the energy consumed. The Cache size and Cache Expiry time are two parameter for an optimization problem and techniques. We use Genetic Algorithms (GA) is used to solve it. After the optimization routing overhead, end to end delay and route discovery time may be reduces.
\end{abstract}

\section{Keywords}

Ad-hoc Wireless Networks, Genetic Algorithm, Quality of Service Requirement, Cache Size and Cache Expiry time.

\section{INTRODUCTION}

The Dynamic Source Routing (DSR) protocol is the protocol of choice in our thesis. It is a simple but very effective routing protocol for MANET. In this section we intend to take a closer look at some of the peculiar features of DSR protocol with regards to it caching mechanism., in order to generate a network with the desired properties while reducing node energy consumption and/or increasing network capacity [1]. Topology Control is required in adhoc wireless networks because there is no central infrastructure to ensure communications. This means that the nodes themselves have the responsibility of ensuring communication. Topology Control provides an effective way of achieving this goal. By using TC, a network topology of the nodes can be constructed which will have the desired properties (in this case meet the QoS requirements) and reduce the node energy consumption and interference between nodes. The topology of the network can be controlled by controlling which links are allowed to be present or absent in the network. In a wireless network, a link is present between two nodes, if both of them are within range of each other. Thus in this case topology control boils down to controlling the transmitting ranges i.e., the transmitting power of the nodes.In this paper, we presented optimization of DSR where the objective is to minimize the overall energy consumed by all the nodes in the network and the constraints are the QoS requirements and interference.

A QoS requirement is a Service Level Agreement (SLA) between a pair of nodes to maintain certain values of communication parameters like bandwidth, delay, hop count etc., The QoS requirements considered in this paper are reduses routing overhead, end to end delay and route discovery time.

\section{RELATED WORK}

This paper is mainly an extension of the work of D. Johnson and Y. Hu., [1] explain caching strategies theoretically. i.e. how caching mechanism store the route in it.

In [2], the authors provide a way of multicast routing between multiple hosts on a network which meets the QoS requirements such as end to end delay and bandwidth requirements. They propose a multi-cast tree selection algorithm based on non-dominated sorting technique of genetic algorithm to simultaneously optimize multiple QoS parameters.In [3], the performance of the DSR caching strategy and it shows how interference on the throughput of wireless networks is studied.

In [4] author proposes a distributed adaptive cache update algorithm for the Dynamic Source Routing Protocol.In [5], the authors show how Cache Invalidation Mechanism for Dynamic Source Routing (DSR) affects the Ad Hoc Networks.

\section{CACHING MECHANISM OF DSR}

One major advantage of DSR protocol is the availability of a caching mechanism. A cache is basically a buffer specifically for storing routes. The caching mechanism in DSR ensures the avoidance of wasteful route discoveries, thereby reducing control overheads and at the same time saving enormous bandwidth and energy. When routes are discovered, they are immediately stored in the nodes ${ }^{\text {ee }}$ routes caches so that subsequent transmission of data packets along the same routes can take place without reinitiating a fresh route discovery process.

One basic design choice to be made in developing a caching strategy for DSR protocol is to determine how the cache is to be organized or structured, i.e., determining the kind of data structure to be used to represent the cache. Two types of cache organization can be used in DSR namely; path cache and link cache. In path cache, a node caches a complete path from route discovery process whereas in link cache, a node caches each link separately. A path cache is not very complex to implement and easily ensures that all paths are loop-free, since each individual route from an RREP is loopfree. To locate a route in a path cache, the source node can simply search its cache for any available path that leads to the destination node. Conversely, to locate a route in link cache, a node must utilise a much more complex search algorithm to locate the current best route through the graph to the destination node. Implementing such search algorithm is very difficult and might involve much CPU processing. Cache capacity is the amount of routes that can be stored in the cache of any particular node. Cache capacity is an important choice to be considered while designing a DSR protocol. For a link cache, the obvious design choice is to allow the cache to store any links that are discovered, since there is a fixed maximum number of N2 links could exist in a 
mobile ad hoc network of $\mathrm{N}$ nodes. However, for a path cache, the maximum storage space that could be needed is much larger than that of link cache, since each path is cached separately and there is no sharing in the data structure even when two paths share a number of common routes. Cache timeout is a strategy devised to deal with the route staleness in DSR caching mechanism. It is the amount of time that a route would remain in a nodees route cache before it would be deleted. Cache timeout predicts the life time of any particular route. Cache timeout strategy, as with cache capacity, also introduces some design choices to be considered while implementing the DSR protocol. Although, a path cache has a mechanism for removing route entries via a capacity limit, for link cache, the timeout can be either static or adaptive. In adaptive timeout, each link is removed from the cache after a certain amount of time has elapsed since the link was added to the cache. On the other hand, in adaptive timeout, a node decides an appropriate timeout after which an added link will be deleted from the cache. The adaptive timeout value should be based on the properties of the link or the nodes constituting the endpoints of the link.

\section{OPTIMIZATION USING GENETIC ALGORITHM}

A lot of optimizations have been proposed for the DSR protocol. There are optimizations to route discovery, route maintenance and caching strategies for the dynamic source routing protocol.

\subsection{Optimization to Route Discovery}

4.1.1 Non-propagating Route Request (RREQ): This optimization attempts to avoid the initiation of a request flooding when the destination of the packets is within the range of the source. To achieve this, a source searching a route to a destination, broadcasts an route request RREQ with the limit of propagation set to zero. In this way, the neighbor nodes receiving this RREQ packet are not allowed to re-broadcast it. After a certain time approx $30 \mathrm{~ms}$, if the source does not receive an RREP packet, it re-broadcasts an RREQ with the limit of propagation set to the maximum value.

\subsubsection{Replying from Cache}

a node receiving an RREQ packet to a destination to which it has a route maintained in its cache, is able to send an Route Reply (RREP) to the source of the RREQ message, instead of rebroadcasting the RREQ packet.

\subsubsection{Gratuitous RREPs:}

This optimization supposes that nodes are on promiscuous mode. When a node overhears a transmission of a packet between two nodes and finds its address in the source route of the packet being transmitted, it can send a GRATUITOUS ROUTE REPLY (GRREP) to the originator of the packet with a shorter route from the source to itself. The source of the packet can store this shorter route in its cache and use it to send subsequent data packets. If this route breaks, the source can reuse the previous route, since it can store more than one route to the same destination.

\subsection{Optimization to Route Maintenance}

\subsubsection{Salvaging:}

when an intermediate node detects that the next hop in the source route is unreachable and it can forward the packet, it attempts to find an alternative route in its cache. If it finds a route to the destination in its cache, it changes the source route of the packet's header and sends the packet using the alternative route. If it does not find a route to the destination, it discards the packet and does not initiate a route discovery process. In both cases, the node sends an RERR message to the source of the data packet.

\subsubsection{Gratuitous Route Errors}

a source receives an RERR message indicating that a route of the source route is broken. If the source broadcasts an RREQ packet, a neighbor not belonging to the source route of the previous RERR might send an RREP with a new source route, but containing the broken path. To avoid this, the source piggybacks this RERR message on its RREQ packet. Thus, the neighbors do not generate RREPs containing the broken path.

\subsection{Optimization to Caching Strategies}

\subsubsection{Snooping}

when a node forwards a data packet, it adds the route from the source to itself. In this optimisation, it can as well "snoop" in the unprocessed part of the source route and add to its cache, the route from itself to the destination of the data packet.

\subsubsection{Tapping}

Nodes operate in promiscuous modes. Under promiscuous mode, nodes disable the address filtering function, thus they receive every packet that they can overhear. They extract important information from these overheard packets, such as new routes from RREPs or information about broken routes from RERRs.

\section{SIMULATION MODELS AND ASSUMPTIONS}

Here we use OPNET simulator and consider the scenario of School of Engineering, Vikram University, Ujjain campus of size 1000 x1000 Sq. Meter for our simulation. We arrange nodes in the scenario and set there attributes likes Name of the node, IP address of the nodes, Subnet Mask etc. After arranging all nodes in the scenario we go through the ADHOC routing parameters from which we choose DSR routing protocol. In DSR routing protocol we specify route cache parameter in bytes and route expiry time in seconds. Here we assume that MAC protocol. During the route discovery procedure, routers maintain ID lists of the recently seen requests to avoid repeatedly processing the same route request. Requests are discarded if they were processed recently since they are assumed to be duplicates. If a router receives a request and detects that the request contains its own ID in the list of intermediate routers, this router discards the request to avoid loops. The route maintenance procedure is used when routes become invalid due to the unpredictable movement of routers. Each router monitors the links that it uses to forward packets. Once a link is down, a route error packet is immediately sent to the initiator of the associated route. Therefore, the invalid route is quickly discarded .To handle unreliable transmissions of control messages, DSR either relies on the underlying MAC protocol to provide guaranteed delivery or it retransmits control messages for a certain number of times. Since DSR is a reactive protocol, it cannot tell whether a destination is unreachable or the route request is lost. Therefore, it suffers more over-head if the underlying MAC layer does not support guaranteed delivery. This is a common problem for reactive routing protocols because when no reply message is heard, routers with a reactive routing protocol cannot tell the difference between the case of a transmission error and the case of unreachable nodes. Reactive routing protocols try to use extra 
acknowledgements or a small number of retransmissions to solve this problem and, thus, introduce more overhead.

\subsection{Performance Metrics}

Two network metrics and one cache metric were evaluated in our simulation work and they are average packet end-to-end delay, routing overhead and route discovery time.

\subsubsection{Average Packet End to End Delay}

The average packet delay of a network is the average time it takes an application on a source node to generate a packet until the packet is received by the application layer of the destination node. It includes delays that arise as a result of propagation and transmission buffering for the period of the route finding, queuing at the network interface and retransmission at the MAC layer.

\subsubsection{Routing Overhead Traffic}

The routing overhead traffic of a network is the amount of routing packets that is transmitted over the network. The routing overhead determines the scalability of the protocol in the network. It is expressed in bits per second or packet per second. Due to the mobility of the nodes in the MANET, there is always an occurrence of link failure which leads to the initialization of route discovery and route maintenance processes. The broadcast request packets generated by route discovery and the route error packet generated as a result of the link failure; will also increase the overhead packets in the network. The route overhead depicts the effectiveness of ad hoc routing protocols.

\subsubsection{Route Discovery Time}

The time taken for the dynamic source routing protocol (DSR) in the source node to establish a route with the DSR in the destination node is called the route discovery time. The route discovery process starts by transmitting a broadcasting request packet by the source node. If an intermediate node does not have the route to the destination node in its route cache, the request packet is retransmitted and at the same time the node will add its address to the route record of the broadcast packet. This is repeated in MANET until the broadcast packet gets to the destination node. The destination will now send the accumulated route to the source node. The time taken to establish this connection in the route.

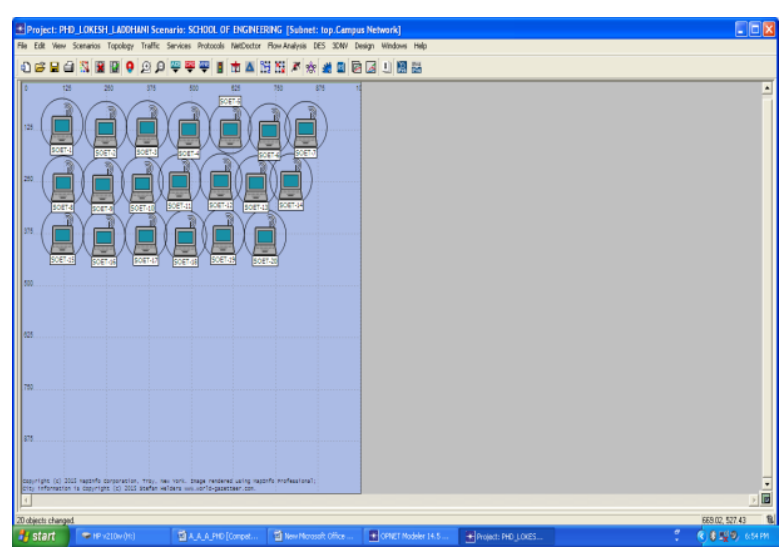

FIG .1 Scenario of 20 mobile nodes in the of SCHOOL OF ENGINEERING USING OPNET 14.5 MODELLER

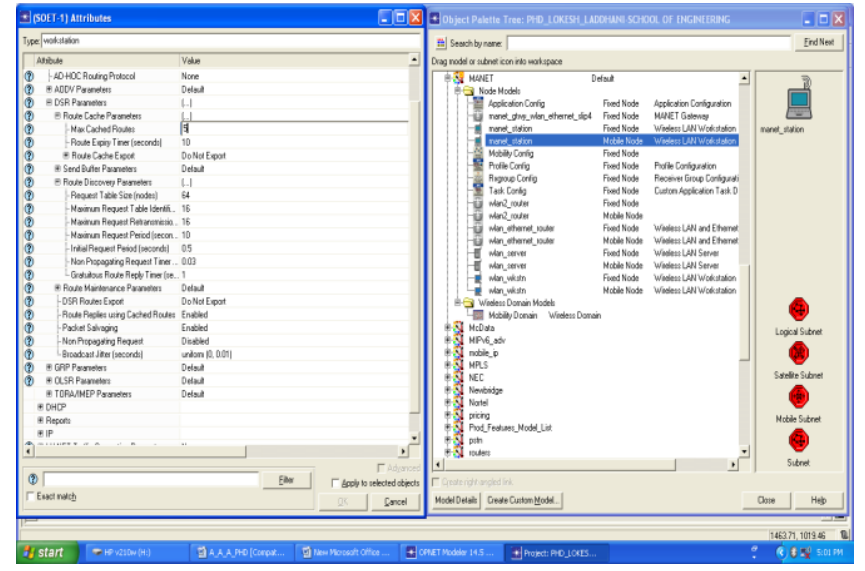

\section{FIG.2 Cache Size 5 bits Route Expiry Time 10 Sec. for 20 nodes\& 30 nodes}

Similar Scenario is made for remaining setups of 20 node and 30 nodes where cache size varies from 5 to 15 bits and route expiry time $10 \mathrm{sec}, 5 \mathrm{sec}$ and $15 \mathrm{sec}$. Below Table shows the results of Simulation done on DSR.

\section{Table- 1Results After Simulation}

\begin{tabular}{|c|c|c|c|c|c|c|c|c|}
\hline $\begin{array}{l}\text { No. } \\
\text { of } \\
\text { No } \\
\text { de }\end{array}$ & $\begin{array}{l}\text { Cach } \\
\text { e } \\
\text { Size } \\
\text { in } \\
\text { bits }\end{array}$ & $\begin{array}{l}\text { Rout } \\
\text { e } \\
\text { Expir } \\
\text { y } \\
\text { Time } \\
\text { in } \\
\text { secs } \\
\end{array}$ & $\begin{array}{l}\text { Ro } \\
\text { uti } \\
\text { ng } \\
\text { Ov } \\
\text { erh } \\
\text { ead } \\
\text { s } \\
\text { in } \\
\text { bits }\end{array}$ & $\begin{array}{l}\text { End to } \\
\text { end } \\
\text { Delay } \\
\text { in secs }\end{array}$ & $\begin{array}{l}\text { Route } \\
\text { Discov } \\
\text { ery } \\
\text { Time } \\
\text { in secs }\end{array}$ & $\begin{array}{l}\text { Mean } \\
\text { Of } \\
\text { Routing } \\
\text { overhea } \\
\text { ds }\end{array}$ & $\begin{array}{l}\text { Coefficient } \\
\text { of } \\
\text { correlation } \\
\text { between } \\
\text { cache size } \\
\text { and } \\
\text { routing } \\
\text { overhead }\end{array}$ & $\begin{array}{l}\text { Coefficien } \\
\mathrm{t} \text { of } \\
\text { correlatio } \\
\mathrm{n} \text { between } \\
\text { cache size } \\
\text { and route } \\
\text { discovery } \\
\text { time }\end{array}$ \\
\hline 20 & 5 & 10 & $\begin{array}{l}246 \\
0\end{array}$ & 0.0037 & 0.101 & \multirow[t]{3}{*}{1641.66} & \multirow[b]{3}{*}{0.99} & \multirow[b]{3}{*}{0.98} \\
\hline 20 & 10 & 10 & $\begin{array}{l}159 \\
7\end{array}$ & 0.0045 & 0.1244 & & & \\
\hline 20 & 15 & 10 & 868 & $\begin{array}{l}0.0062 \\
7 \\
7\end{array}$ & 0.1705 & & & \\
\hline 20 & 10 & 5 & $\begin{array}{l}160 \\
0\end{array}$ & $\begin{array}{l}0.0049 \\
1 \\
\end{array}$ & 0.1241 & 1592 & \multirow{3}{*}{0} & \multirow{3}{*}{0} \\
\hline 20 & 10 & 10 & $\begin{array}{l}159 \\
6\end{array}$ & $\begin{array}{l}0.0045 \\
5\end{array}$ & 0.1248 & & & \\
\hline 20 & 10 & 15 & $\begin{array}{l}158 \\
0 \\
\end{array}$ & $\begin{array}{l}0.0043 \\
4 \\
\end{array}$ & 0.1271 & & & \\
\hline 30 & 5 & 10 & $\begin{array}{l}371 \\
6\end{array}$ & 0.0076 & $\begin{array}{l}0.0778 \\
5\end{array}$ & \multirow[t]{3}{*}{3060} & \multirow[t]{3}{*}{0.99} & \multirow[t]{3}{*}{0.98} \\
\hline 30 & 10 & 10 & $\begin{array}{l}311 \\
7\end{array}$ & $\begin{array}{l}0.0076 \\
3\end{array}$ & $\begin{array}{l}0.0852 \\
2\end{array}$ & & & \\
\hline 30 & 15 & 10 & $\begin{array}{l}234 \\
7 \\
\end{array}$ & 0.0084 & 0.1028 & & & \\
\hline 30 & 10 & 5 & $\begin{array}{l}312 \\
7\end{array}$ & $\begin{array}{l}0.0076 \\
1\end{array}$ & $\begin{array}{l}0.0841 \\
5\end{array}$ & \multirow[t]{3}{*}{3120} & \multirow[t]{3}{*}{0} & \multirow[t]{3}{*}{0} \\
\hline 30 & 10 & 10 & 311 & 0.0076 & 0.0852 & & & \\
\hline 30 & 10 & 15 & $\begin{array}{l}311 \\
5\end{array}$ & $\begin{array}{l}0.0076 \\
1\end{array}$ & $\begin{array}{l}0.0856 \\
2\end{array}$ & & & \\
\hline & & & & & & & & \\
\hline
\end{tabular}

\section{DISSCUSSION}

In above simulation on 20 nodes and 30 nodes shows that as when we increase the cache size parameter and set the route expiry time constant the routing overhead reduces. In the same scenario when we increase the number of nodes there is no change in the result. In the next simulation we set the parameter of the cache size kept constant and vary route expiry time there is no visible impact on the routing overheads. So the above tables demonstrate that higher the cache size the more discover routes can be accommodated in it which in turn reduces the overheads occurs during discovery of new routes. We also find some other mathematical results which justify our findings i.e. coefficient of correlation between the cache size with routing overheads and route discovery time which are highly correlated with each other. 


\section{CONCLUSION}

Hence the energy consumption in finding the routes is optimized and resources are well utilized. It can also be concluded that the performance of the Dynamic Source Routing protocol is dependent on the cache size and not the cache expiry time (cache timeout). This implies that a path cache has got a special mechanism that deletes route entries through a cache capacity limit. It should be noted that the cache timeout is the maximum amount of time when a particular route can remain in the cache of a node before being deleted. This generalization comes in handy when considering design choices for the dynamic source routing protocol.

\section{REFERENCES}

[1] D. Johnson, D. Maltz and Y. Hu. "The Dynamic Source Routing Protocol (DSR) for Mobile Ad Hoc Networks forIPv4",IETFRFC4728.http://www.ietf.org/rfc/rfc4728. txt?number=4728, accessed April 2007.

[2] Abhishek Roy, Nilanjan Banerjee, Sajal K. Das, "An efficient multi objective QoS-routing algorithm for wireless multicasting", Vehicular Technology Conference, VTC Spring 2002, IEEE 55 ${ }^{\text {th }}$, vol. 3, pp. 1160-1164, August 2002.
[3] M. Marina and S. Das. "Performance of Route Caching Strategies in Dynamic Source Routing", In Proceedings of IEEE International Workshop on Distributed Computing Systems, pp. 425 - 432, Mesa Arizona, 2001.

[4] X. Yu and Z. Kedem. "A Distributed Adaptive Cache Update Algorithm for the Dynamic Source Routing Protocol", In Proceedings of the 24th Joint Conference (INFOCOM 2005) of the IEEE Computer and Communications Societies, Vol. 1, pp. 730-739, 2005.

[5] J. Garrido and M. Marandin. "A Link Cache Invalidation Mechanism for Dynamic Source Routing (DSR) in Ad Hoc Networks", in Proceedings of IEEE 18th International Symposium on Personal, Indoor and Mobile Radio Communications, pp. 1 -5, 2007.

[6] L. Laddhani and Umesh Singh "Optimization of Dynamic Source Routing Protocol Considering the Mobility Effect of Nodes in Cache parameter using OPNET", in International Journal of Scientific and Engineering Research, Vol.6, Issue.6 August 2015. 